\title{
Gasto energético em adultos do município de Niterói, Rio de Janeiro: resultados da Pesquisa de Nutrição, Atividade Física e Saúde - PNAFS
}

\author{
Energy expenditure of adults in the city of Niterói, state of Rio de \\ Janeiro: Nutrition, Physical activity and Health Survey - PNAFS
}

Luiz Antonio dos Anjos 1,2

Bianca Catarina M iranda Ferreira ${ }^{1}$

$M$ auricio Teixeira Leite de Vasconcellos ${ }^{3}$

Vivian Wahrlich ${ }^{1}$

${ }^{1}$ Laboratório deAvaliação Nutricional eFuncional (LANUFF), Departamento deNutrição Social,

UniversidadeFederal

Fluminense. Rua M ario

Santos Braga 30/415,

Campus do Valonguinho.

24020-140 Niterói RJ.

anjos@ensp.fiocruz.br

2Escola Nacional deSaúde

Pública Sergio Arouca,

Fundação Oswaldo Cruz.

${ }^{3}$ Escola $\mathrm{N}$ acional de

Ciências Estatísticas

Fundação Instituto

Brasileiro de Geografia e

Estatística.
Abstract The accurate assessment of energy expenditure (EE) and of the physical activity level (PAL) is important for establishing the energy requirements (ER) of populations. Little is known about these variables in the Brazilian population. The purpose of the present study was to assess $\mathrm{EE}$ and PAL in the adult population ( $\geq 20$ years) of Niterói, RJ. An adapted version of the M OSPA time-budget questionnaire was used to assess the duration of the daily activities of the subjects. The energy cost of the activities was obtained from the tablepublished by FAO in 2004, expressed as multiples of the basal metabolic rate (BM R) measured by indirect calorimetry. Total daily EE (TDEE) was calculated as the sum of EE of all activities of a typical daily routine. TDEE was higher in males than in females $(2382.0 \pm 38.0$ and $1987.1 \pm 22.9$ kcal.day ${ }^{1}$ respectively) but women showed higher PAL values $(1.70 \pm 0.02$ and $1.75 \pm 0.01$ respectively). ER estimated using a PAL of 1.40 was the best predictor of $E E$ of the population in all nutritional status categories, particularly for males. In conclusion, it seems prudent to use lower PAL values when estimating the ER of the adult population of Niterói. It is also evident that more data on the energy cost of activities must be generated for establishing the ER of the Brazilian population. Key words Energy requirements, Physical activity level, Adult, Epidemiology
Resumo Conhecer o gasto energético (GE) e 0 nível de atividade física (NAF) serve como base para o estabelecimento dos requerimentos energé ticos (RE) de populações. Pouco se conhece sobre o GE da população brasileira, lacuna que deve ser preenchida visto o quadro de transição nutricional presenteno país. Assim, 0 objetivo destetrabaIho foi avaliar o GE eo NAF da população adulta ( $\geq 20$ anos) do município de Niterói, RJ. Para a obtenção do tempo de duração das atividades cotidianas, usou-se um questionário de orçamento do tempo das atividades cotidianas. 0 custo energético (CE) das atividadesfoi obtido na tabela da FAO de 2004 como múltiplo da taxa metabólica basal (TM B). 0 GE total diário (GETD) foi computado como o somatório do GE de todas as atividades de um dia típico. O GETD foi maior nos homens $(2382,0 \pm 38,0)$ do que nas mulheres $(1987,1+$ $\left.22,9 \mathrm{kcal} . \mathrm{dia}^{-1}\right)$ masasmulheresapresentaram N AF maiores. 0 RE usando o valor de N AF de 1,40 foi 0 que melhor estimou o GETD na população em todas as categorias de estado nutricional. Parece ser prudenteutilizar valores menores de NAF para a estimativa dos RE na população adulta de $\mathrm{N}$ iterói. Fica evidente a necessidade de se obter mais dados deCE deatividades para, junto com estimativas adequadas da TM B, se poder determinar os RE da população brasileira.

Palavras-chave Requerimento energético, Nível de atividade física, Adulto, Epidemiologia 
Introdução

Ao longo das últimas décadas, o crescente processo demecanização eautomação influenciou de forma significativa o estilo de vida, causando mudanças no padrão de atividade física (AF) e nos hábitos alimentares em populações de várias regiões do mundo ${ }^{1}$. Este fenômeno, relacionado diretamente com mudanças econômicas, ambientais, demográficas e culturais, vem acompanhado de aumento na incidência de obesidade com concomitante redução do baixo peso, caracterizando um quadro de transição nutricional².

A redução na realização de atividade física é fator predisponente à obesidade, que aparece como o resultado do desequilíbrio entrea ingestão e o gasto energético (GE) ${ }^{3}$. A obesidade, junto com a diminuição da atividade física, predispõe os indivíduos ao desenvolvimento de doenças crônicas não transmissíveis, particularmente a doença coronariana, a hipertensão arterial sistêmica, o diabetes do tipo II e a osteoporose 4 . Portanto, a determinação, de forma acurada, do nível e da intensidade da AF, que são expressos em função do $G E$, vem se tornando indispensável em estudos epidemiológicos.

A partir de 1985, passou-sea orientar o cálculo dos requerimentos energéticos (RE) de populações através da estimativa do GE total diário ${ }^{5}$, procedimento que foi confirmado na mais recenterecomendação da FAO/WH O/U NU ${ }^{6}$, em 2004. Para tanto, obtém-se o tempo de realização de todas as atividades durante um dia típico, ou seja, faz-se uma avaliação do orçamento do tempo. Para cada atividade, obtém-se o custo energético das atividades, fornecidas por tabelas de conversão deatividade/dispêndio energético, queémultiplicado pelo tempo realizando a atividade, chegando-se, ao se somar o GE das 24 horas, ao GE total diário. A esse procedimento chama-se de método fatorial da determinação dos RE.

Astabelas de conversão, em sua maioria, apresentam os valores das atividades em quilocalorias por minuto ( $\left.\mathrm{kcal} . \mathrm{min}^{-1}\right)$, sem considerar, em geral, dados como gênero, composição corporal, aptidão física e idade. $\mathrm{Na}$ tentativa de contornar al guns desses fatores, passou-se a expressar o GE das atividades como múltiplo da taxa metabólica basal (TMB), ou seja, em unidades de razão de atividade física (RAF). U ma forma simplificada do método fatorial preconiza a multiplicação da TMB de 24 horas pelo nível de atividadefísica (NAF=GE de 24h / TM B de 24h), que édefinido em três níveis dependendo do estilo de vida dos indivíduos ${ }^{6}$ : leve ou sedentário
(1,40 a 1,69); moderado ou ativo $(1,70$ a 1,99) e intenso ou pesado $(2,00$ a 2,40$)$.

$\mathrm{Na}$ prática clínica ou em estudos epidemiológicos, a avaliação da TM B, epor conseqüência o GE, é estimada por equações de predição que, para segmentos da população brasileira, já foram documentadas como inadequadas ${ }^{7-9}$. 0 objetivo do presente estudo foi calcular o GE, uma estimativa dos RE e o NAF para a população adulta do município de Niterói, RJ.

\section{Métodos}

0 presente estudo, de caráter transversal, foi realizado no período dejaneiro a dezembro de 2003 em uma amostra probabilística da população adulta ( $\geq 20$ anos de idade) do município de $\mathrm{Ni}$ terói, estado do Rio de Janeiro - a Pesquisa de Nutrição, Atividade Física e Saúde (PNAFS). A amostra foi selecionada em três estágios, com base no Censo Demográfico $2000^{10}$. No primeiro estágio, os setores do censo foram ordenados segundo renda média domiciliar para, de forma implícita, estratificar por renda e garantir a presença de todos os níveis de renda na amostra, que foi composta por 22 setores para cada quinto de renda. Os valores de cada quinto correspondem ao total do rendimento nominal mensal do responsável pelo domicílio, calculado como o somatório do rendimento mensal detrabalho com o rendimento proveniente de outras fontes. $\mathrm{N}$ a seqüência, os setores censitários foram selecionados de cada estrato de forma sistemática, com probabilidade proporcional a uma medida de tamanho, definida a partir do número de domicílios particulares permanentes de cada setor. Esta etapa foi encerrada ao serem selecionados 110 setores censitários.

No segundo estágio, em cada setor selecionado, dezesseis domicílios foram selecionados com eqüiprobabilidade usando o procedimento de amostragem inversa ${ }^{11}$, conduzindo a um tamanho de amostra de 1.760 domicílios. As listagens dos setores do Censo D emográfico 2000 serviram de cadastro de seleção de domicílios nos 110 setores selecionados no primeiro estágio, sendo os números de ordem dos domicílios na referida listagem selecionados para estabelecer a ordem devisita. Os domicíliosforam, então, visitados na ordem preestabelecida na sel eção e os resultados de cada visita foram registrados em instrumento de coleta próprio. As visitas terminavam quando fosse obtida a décima sexta entrevista realizada no setor, obedecendo aos critérios 
de elegibilidade estabelecidos para a PNAFS, que incluíam a aceitação da pesquisa por um morador adulto ( $\geq 20$ anos de idade), como descrito adiante.

No terceiro estágio, foi selecionado um adulto em cada domicílio entrevistado, dando igual probabilidade de seleção a todos os moradores adultos do domicílio. Este adulto deveria ser elegível para ter a freqüência cardíaca monitorada, ou seja, sua participação estava condicionada a não apresentar qualquer situação clínica que pudesseinfluenciar o metabolismo: utilização de medicamento que alterasse a frequêencia cardíaca ou metabolismo, realização de dieta alimentar, assim como estar no período de gestação ou lactação. Assim, o terceiro estágio representa um viés de seleção do domicílio, uma vez que só puderam participar da pesquisa aqueles domicílios nos quais pelo menos um adulto fosse elegível. Se o viés de seleção de domicílios com adultos saudáveis não pôde ser corrigido, o viés relacionado à distribuição por gênero e idade da população de Niterói, comum em qualquer pesquisa domiciliar, pôde ser corrigido por meio da calibração dos pesos amostrais. 0 peso amostral foi calculado pelo produto do inverso das probabilidades de seleção em cada estágio e a calibração dos pesos amostrais foi feita pela técnica Integrated household weighting system, que assegura a coerência das estimativas com totais populacionais disponíveis para pós-estratos constituídos, no caso, por gênero e grupos etários ${ }^{12}$.

0 adulto sorteado de cada domicílio foi convidado a comparecer ao Laboratório de Avaliação Nutricional e Funcional (LANUFF) para a realização de medições antropométricas e metabólicas. Um total de 548 adultos residentes no município de Niterói, sendo 198 homens e 350 mulheres, realizaram esses procedimentos. A visita ao LANU FF era agendada pela manhã, quando foram realizadas as medidas após os sujeitos assinarem o termo de consentimento livre e esclarecido. A massa corporal foi aferida em balança eletrônica (TAN ITA TBF 305) com precisão de $0,2 \mathrm{~kg}$, com o sujeito descal ço e trajando roupa padronizada fornecida pelo laboratório.

A estatura foi medida em estadiômetro de madeira seguindo padronização de Lohman et al. ${ }^{13}$. 0 indivíduo foi posicionado no estadiômetro com os braços ao longo do corpo, pés unidos e centralizados no equipamento, cabeça, nádegas e calcanhares encostados no antropômetro, descalços e com os olhos mantidos num plano horizontal (linha de Frankfört). A régua do estadiômetro foi então deslocada até a cabeça do vo- luntário e realizada a leitura após uma expiração normal. Foram feitas duas aferições da estatura e considerou-se a média dos valores para as análises. De posse dos dados de massa corporal e estatura, calculou-se o índice de massa corporal (IM C; Kg.m²), através da divisão da massa corporal $(\mathrm{kg})$ pelo quadrado da estatura $\left(\mathrm{m}^{2}\right)$. Este índice foi utilizado para se determinar o estado nutricional de acordo com a classificação proposta pelo comitê de expertos da Organização Mundial da Saúde ${ }^{14}$.

A medida de TM B foi realizada após as medições antropométricas numa sala tranqüila, sem claridade e ruídos e com temperatura controlada em torno de $25^{\circ} \mathrm{C}$, com o sujeito em jejum de doze horas. Os detal hes da medição podem ser encontrados em outra publicação ${ }^{15}$.

Os participantes da pesquisa também responderam a um questionário que continha informações acerca dos dados pessoais, dados ocupacionais ede AF edados de dieta e desaúde. 0 presente estudo utilizou uma partedestequestionário, proveniente da seção que se dedica a avaliar as atividades realizadas em um dia típico do sujeito, o Questionário de Orçamento do Tempo (QOT - Quadro 1). Esse questionário foi adaptado do questionário utilizado no estudo The M ONICA Optional Study of Physical Activity - M OSPA ${ }^{16}$. O questionário foi desenvolvido pelo Centers for Disease Control and Prevention (CDC) com a assistência de especialistas da área e de indivíduos envolvidos com o estudo M ONICA ${ }^{17}$ e sua validade e confiabilidadejá foram estabelecidas, principalmente no que diz respeito às atividades ocupacionais e no lazer ${ }^{18,19}$. D esde então, estudos em diversos países, como Finlândia ${ }^{20}$, Suécia ${ }^{21}$, Paquistão ${ }^{18}$, Bélgica ${ }^{19}$ eChina ${ }^{22}$ vêm utilizando versões do M OSPA para avaliar padrões de atividadefísica e GE de grupos populacionais. A adaptação para o presente estudo foi simplesmente usar um dia típico como referência para as respostas e a retirada da estimativa da intensidade dada pela alteração da ventilação.

O GE foi calculado para cada atividade descrita multiplicando-seo valor de RAF (GE minuto / TMB minuto) extraído da tabela de gasto energético das atividades da Food and Agriculture Organization of the U nited Nations (FAO) ${ }^{6}$ pelo tempo de duração da atividade do QOT e pela TMB minuto estimada através da equação de Schofield, ${ }^{23}$ recomendada para uso internacio$\mathrm{nal}^{6}$, e pela TM B medida. Os valores de RAF usados para homens e mulheres, respectivamente, foram $6: 1,4$ e 1,5 para o tempo sentado ou de pé no trabalho; 2,8 e 3,0 para a variável andando no 
Quadro 1. M odelo do questionário de orçamento do tempo (QOT) utilizado na Pesquisa de Nutrição, Atividade Física e Saúde - PNAFS, 2003.

Questionário de orçamento do tempo
1. Trabalhando (todas atividades remuneradas ou voluntárias)
2. Sentado ou em pé no trabalho
3. Andando no trabalho
4. Levantando ou carregando objetos de até 10 kg no trabalho
(ou realizando atividade de esforço semelhante)
5. Levantando ou carregando objetos de > 10 kg no trabalho
(ou realizando atividade de esforço semelhante)
6. Indo e voltando (ida + volta) do trabalho
7. Andando (fora do trabalho, incluindo lazer e excluindo trabalho)
8. Realizando tarefas domésticas intensas
(exemplo: varrer e esfregar chão, lavar roupa a mão)
9. Realizando outras tarefas não intensas em casa
10. ealizando outras atividades sociais ou de lazer
(participação em associações, cultos religiosos etc)
11. Dormindo
12. Exercício para condicoinmento físico. Qual?
14. Assisindo TV sentado, trabal hando no computador
(incluindo internet) ou brincando em vide-games
13. Outras atividades:
(a)

ou indo para, e voltando do, trabalho; 3,5 para o tempo levantando ou carregando objetos de até $10 \mathrm{~kg}$ no trabalho e 3,9 quando levantando ou carregando objetos com mais de $10 \mathrm{~kg}$ no trabaIho; 2,0 para o deslocamento de carro, 1,2 para os deslocamentos detrem, ônibus, barca ou me trô, 5,6 e 3,6 para deslocamento de bicicleta, 2,7 para deslocamento de motocicleta; 2,8 para tarefas domésticas intensas e 2,3 para as outras tarefas não intensas em casa; 1,2 para outras atividades sociais ou de lazer; 1,0 para o tempo dormindo; e 1,4 para assistir TV, usar o computador ou jogar videogames. Asvariáveis exercício paracondicionamento físico e outras atividades, que foram perguntadas no formato aberto, geraram uma extensa lista de atividades cujos valores de RAF foram atribuídos assumindo-se valores para atividades semelhantes quando não estavam disponíveis na tabela. Para as atividades sem valores de RAF na tabela para homens (ou muIheres), o valor das mulheres (ou dos homens) foi imputado universalmente. No caso em que 0 somatório do tempo do dia típico não tenha alcançado as 24 horas, utilizou-se 0 valor de RAF 
de 1,4 para esse tempo residual que foi no máximo de noventa minutos.

$O$ GE total das 24 horas foi obtido somando-se todos os GE de atividades usando a TM B estimada $\left(G_{E_{\text {st }}}\right.$ ) ou medida $\left(G E_{\text {Med }}\right.$ ). 0 nível de atividade física (NAF) foi obtido dividindo-se o $\mathrm{GE}_{\mathrm{Med}}$ pela TM B de 24 horas medida classificando 0 estilo de vida segundo as faixas sugeridas pela FAO/WH O/U NU ${ }^{6}$ como: leve ou sedentário $(1,40$ a 1,69$)$; moderado ou ativo $(1,70$ a 1,99$)$ e intenso ou pesado $(2,00$ a 2,40$)$.

Os valores de GE para a população como um todo também foram estimados seguindo o recomendado pela FAO (FAO/WHO/UNU, 2004) ${ }^{6} \mathrm{e}$ pelo Institute of $\mathrm{M}$ edicine (IOM ) norte-americano, que fornece os dados conhecidos como DRI (recomendações dietéticas) ${ }^{3}$. Para o primeiro, usou-se a estimativa da TM B pela equação de Schofield multiplicado pelo valor mínino decada uma das faixas de NAF estabelecidos para o estilo de vida descrito acima, a saber: leve $(1,4)$, moderado $(1,7)$ e pesado $(2,0)$. Para o segundo, os valores foram obtidos em função do gênero e NAF usando as equações para estimativa do GE total diário sugeridas para uso em populações que tenham tanto indivíduos com IMC dentro da faixa adequada quanto com sobrepeso ou obesidade 3 : homens, GE $=864$ - $(9.72 \times$ idade em anos) +(NAF x (14.2 $\times$ massa corporal em $\mathrm{kg}))+(503 \times$ estatura em $\mathrm{m}$ ) com N AF $=1,12$ (pouco ativo) e 1,27 (ativo) e mulheres, GE = 387(7.31 $\mathrm{x}$ idade em anos) +(NAF $\times(10.9 \times$ massa corporal em $\mathrm{kg}))+(660.7 \times$ estatura em $\mathrm{m}$ ) com $\mathrm{NAF}=1,14$ (pouco ativo) e 1,27 (ativo). $\mathrm{Na}$ comparação do GE por estado nutricional, as equações específicas para os indivíduos com sobrepeso e obesidade foram usadas.

Para análise das informações, foi utilizada estatística descritiva referente às ocorrências, em médias, erros padrão e intervalos de confiança de $95 \%$. As comparações entremédias de GE medido e estimado foram feitas utilizando o método de expansão de Taylor para estimar o erro amostral dos estimadores baseado no desenho amostral complexo. Foram usados os procedimentos Surveymeans e Freq do programa estatístico SAS (Statistical Analysis System, versão 9.1), que trata, adequadamente, desenhos de amostra complexos com o uso de pesos calibrados para representar os 324.671 adultos de Niterói (178.785 mulherese 145.886 homens). Todos os procedimentos da PNAFS foram aprovados pelo Comitê de Ética em Pesquisa da Escola Nacional de Saúde Pública Sergio Arouca da Fundação Oswaldo Cruz.

\section{Resultados}

A idade média ( \pm erro padrão) da população de $N$ iterói foi de $45,0 \pm 0,8$ anos entre as mulheres $e$ $42,6 \pm 1,0$ anos entre os homens e o IM C médio foi de $25,7 \pm 0,3$ e 25,4 $\pm 0,3$, respectivamente (Tabela 1). A prevalência desobrepeso ( $25 \leq$ IM C $<30 \mathrm{~kg} \cdot \mathrm{m}^{-2}$ ) foi semelhante entre homens $(32,9 \%)$ e mulheres $(32,2 \%)$ mas a obesidade $\left(\mathrm{IMC} \geq 30 \mathrm{~kg} \cdot \mathrm{m}^{-2}\right)$ foi levemente superior nas mulheres $(17,5 \%)$ do que nos homens $(13,2 \%)$.

O $\mathrm{GE}_{\text {Med }}$ das mulheres foi, em média, 1987,1 $\pm 22,9 \mathrm{kcal}^{\mathrm{M}}$.dia-1. $\mathrm{O} \mathrm{GE}_{\mathrm{Est}}$ ao ser calculado através da TM B estimada foi de $2361,2+22,8 \mathrm{kcal}$. dia ${ }^{-1} \mathrm{e}$ foi significativamente superior ao $\mathrm{GE}_{\mathrm{Med}}$. $\mathrm{OGE}$ estimado pela equação do IOM para um NAF ativo foi, entre os vários valores de GE estimados, o único que não foi significativamente diferente do $\mathrm{GE}_{\text {Med }}$. $O$ GE estimado pela FAO usando o valor mínimo de NAF para a atividade leve $(1,4)$ e com o método do IOM para os indivíduos pouco ativos forneceu dados inferiores ao do $\mathrm{GE}_{\text {Med }}$ em torno de $100 \mathrm{kcal}^{\text {ddia-1 }}$ (Tabela 2). A média do NAF medido foi de $1,75 \pm 0,01$, ficando sempre dentro do que se considera como estilo de vida moderado $(1,7-1,99)$, com exceção das mulheres mais jovens ( $20-30$ anos, N AF=1,65).

$O \mathrm{GE}_{\mathrm{Med}}$ dos homens foi sempre superior ao das mulheres (Tabela 2). Como para as mulheres, o valor de NAF ficou, em geral, dentro da faixa considerada pela FAO como estilo de vida moderado. $0 \mathrm{GE}_{\mathrm{Est}}$ pelo modelo da FAO usandose o valor mínimo para o estilo de vida leve foi 0 único, entre os valores de GE estimados, que não foi significativamentediferente do $\mathrm{GE}_{\mathrm{Med}}$.

Os valores médios do $\mathrm{GE}_{\mathrm{Med}}$ aumentaram com o aumento do IMC tanto para homens quanto para mulheres de Niterói. As mulheres com baixo peso (IMC < 18,5 kg. $\mathrm{m}^{-2}$ ) apresentaram o menor valor médio do $\mathrm{GE}_{\mathrm{Med}}(1569,1 \pm$ 40,7 kcal. dia-1 ${ }^{-1}$ mas os homens com baixo peso tiveram, de forma surpreendente, um $\mathrm{GE}_{\mathrm{Med}}$ relativamente alto (Figura $1 \mathrm{~A}$ e B). $\mathrm{O} \mathrm{GE} \mathrm{E}_{\mathrm{Est}}$ pela FAO (leve) não foi diferente do $\mathrm{GE}_{\text {Med }}$ para cada categoria de estado nutricional nos homense para as mulheres de baixo peso.

À medida que o IMC das mulheres aumentou, o NAF estimado também sofreu elevação (Figura 2A). No caso dos homens, os indivíduos que apresentaram maior valor de NAF estimado $(1,93 \pm 0,047)$ foram os classificados com baixo peso (IMC $<18,5 \mathrm{~kg} \cdot \mathrm{m}^{-2}$, Figura $2 \mathrm{~B}$ ). Curiosamente, o menor valor de NAF médio entre as mulheres foi encontrado entre as classificadas como de baixo peso, com valor de 1,55 $\pm 0,016$. 
Os homens que apresentaram o menor NAF médio $(1,68 \pm 0,007)$ foram os que apresentaram sobrepeso.

\section{Discussão}

A relação entreinatividade física e doenças crônicas não transmissíveis (DCNT), como doenças cardiovasculares, diabetes tipo II, obesidade, câncer de cólon e osteoporose, na população adulta, é apoiada por fortes evidências ${ }^{24,25}$. Existe, portanto, grandedemanda de se conhecer as caracte- rísticas de atividade física e, como a intensidade da atividade física é definida em função do nível deGE das atividades, a estimativa do GE depopulações vem se transformando numa variável de grande interesse em pesquisas epidemiológicas.

Os dados para calcular o GE na população adulta de Niterói foram obtidos através de um questionário de orçamento de tempo que obtém dados de todas as atividades dos indivíduos ${ }^{16} \mathrm{e}$ que já foi validado e vem sendo usado em diversas partes do mundo ${ }^{18-22}$. A alternativa a esse método seria o estabelecimento dos RE a partir de dados do GE advindos de estudos com água duplamentemarcada (ADM). Esse método, con-

Tabela 1. Estimativa de médias, erro padrão (EP) e intervalo de confiança (IC) da idade e medidas antropométricas da população adulta ( $\geq 20$ anos) de N iterói. Pesquisa de Nutrição, Atividade Física e Saúde - PNAFS, 2003.

\begin{tabular}{lcccccc}
\hline & \multicolumn{4}{c}{ Mulheres } & \multicolumn{3}{c}{ Homens } \\
\hline & M édia & EP & IC 95\% & M édia & EP & IC $95 \%$ \\
\hline Idade (anos) & 45,0 & 0,8 & $43,4-46,6$ & 42,6 & 1,0 & $40,6-44-6$ \\
M assa corporal (kg) & 64,0 & 0,7 & $62,5-65,4$ & 7431 & 1,1 & $72,0-76,3$ \\
Estatura (cm) & 158,5 & 0,3 & $15780-159,2$ & 171,4 & 0,7 & $170,0-172,7$ \\
IM C $\left(\mathrm{kgm}^{-2}\right)^{*}$ & 25,7 & 0,3 & $25,1-26,3$ & 25,4 & 0,3 & $24,8-26,1$ \\
\hline
\end{tabular}

${ }^{*} \mathrm{IM}$ C - Índicedemassa corporal $=$ massa corporal. estatura ${ }^{2}$.

Tabela 2. M édias e erro padrão (EP) do gasto energético (GE) usando a taxa metabólica basal (TM B) medida (GE medido) ou estimada (GE estimado usando TM B estimada), o GE estimado pela IOM e pela FAO e o NAF (GEM ed / TM B medida) segundo o gênero da população adulta ( $\geq 20$ anos) de Niterói. Pesquisa de Nutrição, Atividade Física e Saúde - PN AFS, 2003.

\begin{tabular}{lrrrc}
\hline \multicolumn{1}{c}{ Variável } & \multicolumn{2}{c}{ Mulheres } & \multicolumn{2}{c}{ Homens } \\
\hline & Média & EP & Média & EP \\
\hline GE M edido (kcal.dia ${ }^{-1}$ ) & 1987,1 & 22,9 & 2382,0 & 38,0 \\
N AF & 1,75 & 0,01 & 1,70 & 0,02 \\
GE Estimado (kcal.dia-1) & & & & \\
Usando TM B estimada & 2361,2 & 22,8 & 2903,9 & 43,8 \\
Pela IOM pouco ativo* & 1899,9 & 13,4 & 2491,0 & 21,0 \\
Pela IOM ativo** & 1990,6 & $14,2^{* * *}$ & 2649,0 & 22,8 \\
Mínimo FAO leve & 1892,0 & 12,0 & 2388,9 & 25,4 \\
Mínimo FAO Moderado & 2297,5 & 14,5 & 2900,8 & 30,8 \\
\hline
\end{tabular}

${ }^{*}$ M ulheres $=387-\left(7.31^{*}\right.$ idade, anos $)+\left(1.14^{*}\left(10.9^{*}\right.\right.$ massa corporal, $\left.\left.\mathrm{kg}\right)\right)+\left(660.7^{*}\right.$ estatura, $\left.\mathrm{m}\right)$; H omens $=864-\left(9.72^{*} \mathrm{idade}\right.$, anos $)+\left(1.12^{*}\left(14.2^{*}\right.\right.$ massa corporal, $\left.\left.\mathrm{kg}\right)\right)+\left(503^{*}\right.$ estatura, $\left.\mathrm{m}\right)$

${ }^{*}$ M ulheres $=387-\left(7.31^{*}\right.$ idade, anos $)+\left(1.27^{*}\left(10.9^{*}\right.\right.$ massa corporal, $\left.\left.\mathrm{kg}\right)\right)+\left(660.7^{*}\right.$ estatura, $\left.\mathrm{m}\right)$; H omens $=864-\left(9.72^{*}\right.$ idade, anos $)+\left(1.27^{*}\left(14.2^{*}\right.\right.$ massa corporal, $\left.\left.\mathrm{kg}\right)\right)+\left(503^{*}\right.$ estatura, $\left.\mathrm{m}\right)$

${ }^{* * * *} \mathrm{Não}$ significativamente diferente do GE (medido) dentro do gênero. 
siderado padrão para a estimativa do $\mathrm{GE}$, fornece, entretanto, somente o GE total diário médio de vários dias e ainda é extremamente caro para ser usado em estudos populacionais ${ }^{26}$. De fato, a mais recente recomendação para a população norte-americana, publicada pela IOM (DRI) e usada para comparação no presente estudo, foi baseada em dados de GE usando ADM de somente 433 mulheres e 334 homens adultos com idade acima de dezoito anos 3 .

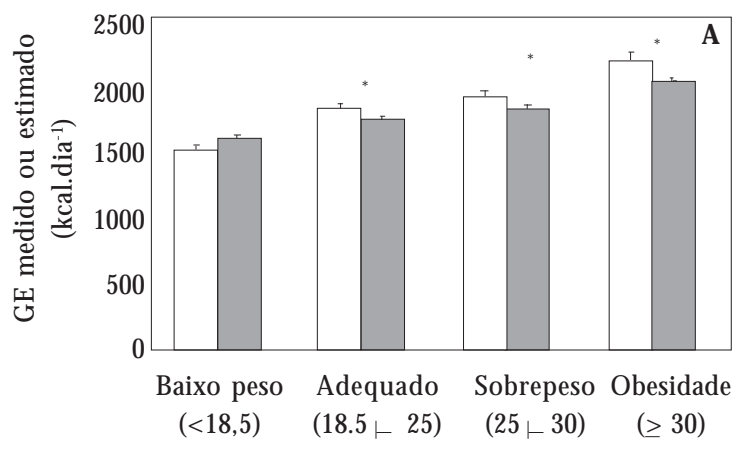

Estado Nutricional (IMC - kg.m-2)

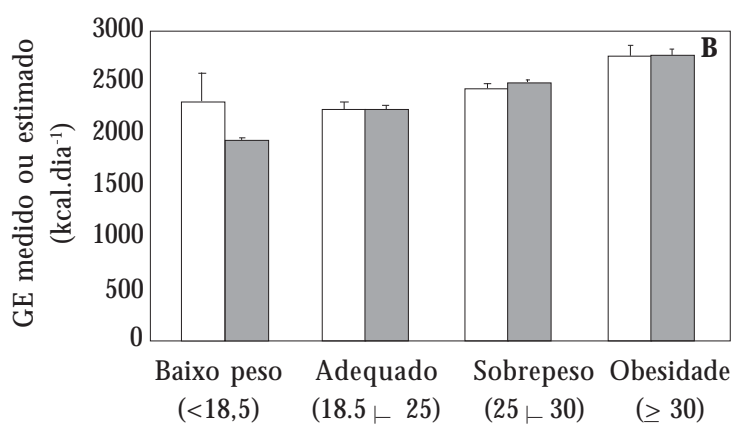

Estado Nutricional (IMC - kg.m-2)

$\square$ M edido
$\square$ Estimado (NAF mínimo leve)

Obs.: 0 asterisco representa diferença significativa $(p<0,05)$.

Figura 1. Valores médios e erro padrão do gasto energético (GE) medido e estimado pelo método da FAO (usando 0 a TM B estimada e o valor mínimo para o NAF leve - 1,4) em relação ao estado nutricional da população adulta ( $\geq 20$ anos) feminina (A) e masculina (B) de Niterói. Pesquisa de Nutrição, Atividade Física e Saúde - PNAFS, 2003.
A estimativa do GE da população de Niterói mostrou que, para as mulheres, o método para calcular o $\mathrm{GE}_{\mathrm{Est}}$ mais adequado, tomando-se por base $0 \mathrm{GE}_{\mathrm{Med}}$ foi a fórmula do IOM (ativo) ${ }^{3}$. Entre os homens, o método descrito pela FAO utilizando o limite inferior do NAF leve $(1,4)$ foi o que mostrou não ser significativamente diferente do $\mathrm{GE}_{\mathrm{Med}}$. Esse método, para as mulheres, apesar de fornecer estimativa de GE significativamente inferior ao $\mathrm{GE}_{\mathrm{Med}^{\prime}}$, forneceu a segunda menor diferença $\left(-95.1 \pm 19.0 \mathrm{kcal}^{\left.-1 \mathrm{dia}^{-1}\right)}\right.$ entreos métodos de estimativa de GE ebem inferior ao se usar a TM B estimada $\left(374.1 \pm 9.4 \mathrm{kcal} \cdot \mathrm{dia}^{-1}\right)$.
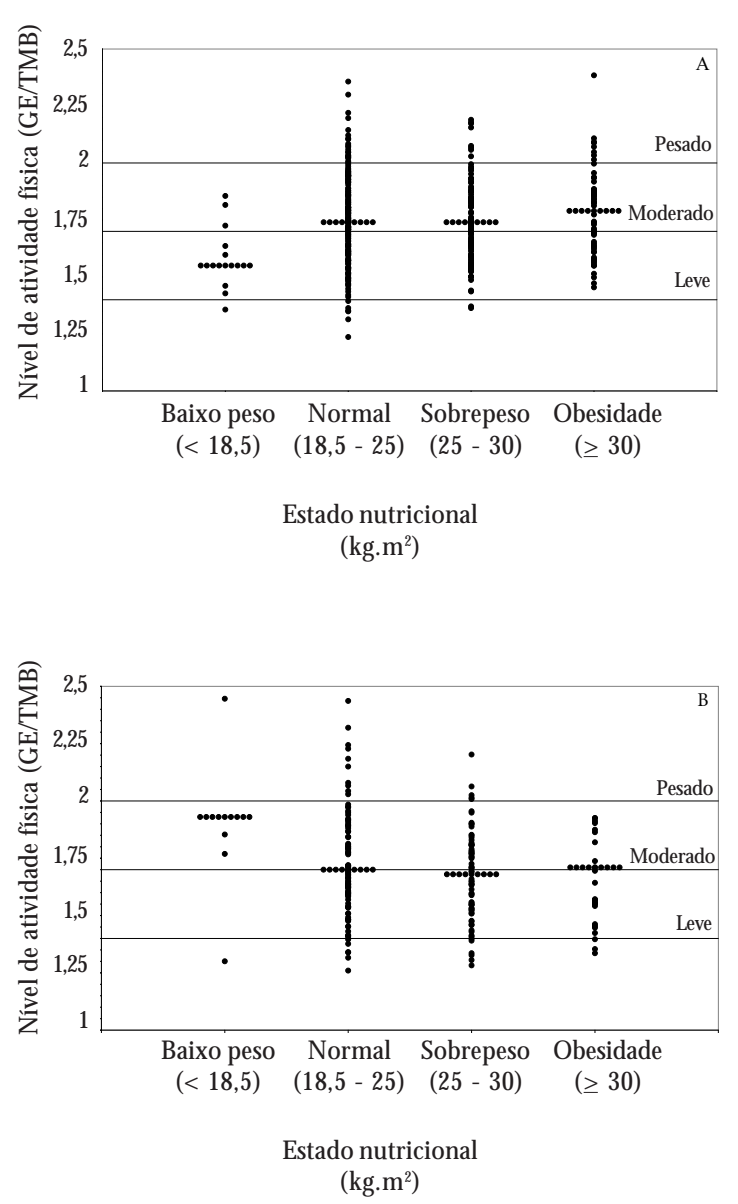

Obs.: 0 asterisco representa diferença significativa $(p<0,05)$. A reta horizontal pontilhadaéa média. As retas inteiras são os pontos de corte para o nível de atividade física (NAF) segundo aFAO/WHO/ UNU (2004).

Figura 2. Valores individuais do nível de atividade física ( gasto energético - GE / taxa metabólica basal - TMB) em relação ao estado nutricional da população adulta ( $\geq 20$ anos) feminina (A) e masculina (B) de Niterói. Pesquisa de Nutrição, Atividade Física e Saúde - PNAFS, 2003. 
Assim, pode-se pensar em sugerir o método da FAO com o limite inferior do NAF leve como uma maneira para o cál culo do RE da população adulta residente em $N$ iterói. Essefato éintrigante, já queo NAF medido tanto em homens quanto em mulheres permitiria classificá-los, segundo a própria tabela da FAO/WHO/UNU ${ }^{6}$, como tendo estilo de vida ativo e, portanto, valores de NAF entre 1,7 e 1,99. De fato, avaliação do padrão de atividade física nessa população indicou que aproximadamente $60 \%$ dos adultos de $\mathrm{Nite}$ rói realizam pelo menos trinta minutos de atividadefísica moderada ${ }^{27}$, recomendação atual para a prevenção, ao nível populacional, de doenças crônicas não transmissíveis e manutenção da saúde ${ }^{28}$. Deve-se lembrar que essas comparações foram feitas com o valor mínimo da faixa de NAF leve eque se o cálculo fosse feito usando-se o valor médio da faixa $(1,55)$, prática corrente entre nutricionistas, o valor de superestimação do GE seria muito maior ainda.

A superestimativa do GE pelo método da FAO, comparativamenteao GE obtido por ADM , já foi documentado em dados de al guns países ${ }^{29}$ mas não em outros ${ }^{22,30}$, o que pode ser explicado pelas diferenças em nível de desenvolvimento, 0 que afetará o padrão de atividade física ocupacional e não ocupacional ${ }^{26}$. Dessa forma, a obtenção das informações sobre o tempo de realização de atividades pode ser um fator de erro na estimativa dos RE. O outro componente, a TM B, deve ser obtido da forma mais acurada possível. A pesar das críticas de que as equações de Schofield ${ }^{23}$ superestimam a TMB em diversas partes do mundo, inclusive no Brasili1, elas foram as recomendadas para uso internacional na mais recente publicação sobre RE da $\mathrm{FAO}^{6}$. Para a população adulta de $\mathrm{N}$ iterói, essa superestimativa chega a aproximadamente $20 \%{ }^{15}$.

0 método de estimativa dos RE recomendado pelo IOM e publicado como as $\mathrm{DRI}^{3}$ é semeIhante ao da $\mathrm{FAO}^{6}$. Ambos baseiam-se nos dados de TM B multiplicados pelo NAF para se estimar o GE. A novidade da mais recente recomendação de energia da FAO é que não mais existe um valor fixo de NAF e sim uma faixa deixando a escolha do valor ao usuário. Como é uma faixa, não há mais sentido em se ter valores distintos para homens e mulheres como no passado. No caso da DRI, escolhe-se um valor único baseado no estilo de vida do indivíduo que também é dado como uma faixa. Com o quadro atual de transição nutricional em que a população brasileira seencontra, com el evação progressiva nas prevalências de sobrepeso e obesidade? a determinação do GE é um procedimento que deve ser cuidadosamente realizado para não se perpetuar esse quadro. A proximadamente metade da população de Niterói mostrou-se com excesso de massa corporal, valores semelhantes aos observados para a população brasileira na mais recente Pesquisa deO rçamento Familiar ${ }^{11,32}$. À medida que o IMC aumentava, o GE medido também apresentava elevação. O GE medido aumenta com o aumento do IM C, tanto em homens quanto em mulheres, fato documentado nas publicações da IOM (DRI) ${ }^{3}$ e FAO ${ }^{6}$, e que significa dizer que indivíduos com sobrepeso e obesidade precisam ingerir mais energia para manter sua maior massa corporal ${ }^{3}$.

Para os homens, o método de se estimar o GE e, portanto, oS RE, da FAO com o valor mínimo de NAF igual a 1,4 mostrou-se adequado para todas as categorias de IMC analisadas. M esmo sendo significativamente diferente para as mulheres, essa estimativa fornece valores de RE menores, o queseria mais prudentepara uma população com alta prevalência de excesso de massa corporal. Já para as mulheres, o método do DRI mostrou-se adequado, fato documentado em outros estudos ${ }^{33}$.

0 crucial atualmente para se estabelecer os RE é se ter boas estimativas dos valores de NAF para populações ${ }^{22,30}$. 0 estabelecimento desses valores vem sendo feito baseando-se em estimativas do GE total diário em estudos usando a técnica da ADM esua divisão pelaTM $B$, quenem sempre é medida. Como a TM B estimada pelas equações de predição parece ser maior do que a medida, questiona-se se os val ores de N AF obtidos nesses estudos podem ser universalizados. Existe uma outra alternativa de se calcular os valores de NAF para uma população e seus segmentos e que, a princípio, não necessita da determinação do GE total diário e nem da TM ${ }^{34}$. 0 NAF pode ser estimado pelas informações de orçamento do tempo, desde que se tenham informações precisas sobre o custo energético (expresso como múltiplo da TMB) das atividades de uma população. Esse tipo de informação é limitada, como pode ser comprovado pela tabe la publicada pela $\mathrm{FAO}^{6}$. Especificamente para a caminhada, nosso laboratório já documentou a validade dos valores tabelados numa amostra de universitárias de $\mathrm{N}$ iterói ${ }^{35}$. Essas informações podem ser obtidas em laboratórios de avaliação funcional com calorímetros indiretos com custo bastante menores do que o necessário em estudos que usam a técnica da ADM. Esses estudos, por outro lado, demandam uma participação 
maior dos sujeitos em termos de número de vindas ao laboratório para as medições de TM B e do custo energético das atividades.

Em suma, os dados do presente estudo pare cem indicar que seja necessário utilizar, para a população adulta deN iterói, valores menores deNAF do que os recomendados. Fica evidente também queénecessário seobter val ores do custo energético das atividades para, junto com equações de TM B adequadas, poder-se estimar com maior acurácia os RE para a população brasileira.

\section{Colaboradores}

LA Anjos eM TL Vasconcellosplanejaram a PNAFS. BCM Ferreira eV Wahrlich coletaram os dados em laboratório. MTL Vasconcellos fez 0 desenho de amostragem e calculou os pesos amostrais naturais e calibrados e realizou as análises junto com LA Anjos. BCM Ferreira e LA Anjos escreveram a maior parte do manuscrito que foi revisado e aprovado por todos autores.

\section{Agradecimentos}

BCM Ferreira recebeu bolsa da Coordenação de Aperfeiçoamento de Pessoal de Nível Superior (CAPES). A PNAFS foi financiada em parte pelo CN Pq (Proc. 471172/2001-4 e 475122/2003-8) e pela Fiocruz (PAPES III - Programa de Apoio a Projetos Estratégicos em Saúde - 250.139). LA Anjos recebeu bolsa de produtividade em pesquisa do CN Pq (Proc. 301076/89-8). M TL Vasconcellos recebeu bolsa de produtividadeem pesquisa do CN Pq (Proc. 302992/2003-0).

\section{Referências}

1. M endonça CP, Anjos LA. Aspectos das práticas alimentares e da atividade física como determinantes do crescimento do sobrepeso/obesidade no Brasil. Cad Saude Publica 2004; 20 (3):698-709.

2. Monteiro CA, Conde WL, Popkin BM. The burden of disease from undernutrition and overnutrition in countries undergoing rapid nutrition transition: A view from Brazil. Am J Public Health 2004; 94(3):433-434.

3. Institute of Medicine of the $\mathrm{N}$ ational Academies. Dietary reference intakes for energy, carbohydrate, fiber, fat, fatty acids, cholesterol, protein, and amino acids. Part 1. Washington: The National Academy Press; 2002.

4. WHO/FIMS. Committee on Physical Activity for Health, 1995. Exercise for health. Bull World Health Organ 1995; 73(2):135-136.

5. FAO (Food and Agriculture Organization) /WHO (World Health Organization) /UNU (United Nations University). Energy and protein requirements. [Technical Report Series 724]. Geneva: WHO; 1985.

6. FAO (Food and Agriculture Organization). Human Energy Requirements. Report of a Joint FAO/WHO/ UNU Expert Consultation. [Technical Report Series 1]. Rome: FAO; 2004.

7. Cruz CM, Silva AF, Anjos LA. A taxa metabólica basal é superestimada pelas equações preditivas em universitárias do Rio de Janeiro, Brasil. Arch Latinoam Nutr 1999; 49(3):232-237.

8. Wahrlich V, Anjos LA. Validação de equações de predição da taxa metabólica basal em mulheres residentes em Porto Alegre, RS. Rev. Saude Publica 2001; 35(1):39-45.

9. Wahrlich V, Anjos LA, Going SB, Lohman TG. Basal metabolic rate of Brazilians living in the Southwestern United States. Eur J Clin Nutr 2007; 61(2):290-294.

10. Fundação Instituto Brasileiro de Geografia e Estatística. Censo Demográfico 2000: agregado por setores censitários dos resultados do universo. 2ª ed. Rio de Janeiro: IBGE; 2003.

11. Bossan FM, Anjos LA, Vasconcellos MTL, Wahrlich V. Nutritional status of the adult population in Niterói, Rio de Janeiro, Brazil: the Nutrition, Physical Activity, and Health Survey. Cad Saúde Pública 2007; 23(8):1867-1876.

12. Silva PLN. Calibration estimation: when and why, how much and how. [Textos para Discussão da Diretoria de Pesquisas 14]. Rio de Janeiro: IBGE; 2004.

13. Lohman TG, Roche AF, Martorell R. Anthropometric standardization reference manual. Champaign, Illinois: Human Kinetics; 1988. 
14. WHO. O besity: preventing and managing the global epidemic, report of a WHO consultation. [WHO Technical Report Series 894]. Geneva: World Health Organization; 2000.

15. Wahrlich V, Anjos LA, Vasconcellos MTL. Basal metabolic rate of Brazilian adults from Niterói, Rio de Janeiro: a population-based study. [Submetido à aprovação].

16. WHO/MONICA. The MONICA Optional Study of Physical Activity (M OSPA). M ed Sci SportsExerc 1997; 29(Suppl 6):S162-169.

17. Kriska AM, Caspersen CJ. Introduction to a collection of physical activity questionnaire. M ed Sci Sports Exerc 1997; 29(Suppl 6):S5-9.

18. Iqbal R, Rafique G, Badruddin S, Qureshi R, GrayDonald K. Validating MOSPA questionnaire for measuring physical activity in Pakistani women. Nutr J 2006; 5:18.

19. Roeykens J, Rogers R, M eeusen R, M agnus L, Borms J, De Meirleir K. Validity and reliability in a Flemish population of the WHO-MONICA Optional Study of Physical Activity Questionnaire. M ed Sci Sports Exerc 1998; 30(7):1071-1075.

20. Barengo NIC, Kastarine M, Lakka T, Nissinen A, Tuomilehto J. Different forms of physical activity and cardiovascular risk factors among 24-64-yearsold men and women in Finland. Eur J Cardiovasc Prev Rehab 2006; 13(1):51-59.

21. Wilhelmsen L, Johansson S, Rosengren A, Wallin I, Dotevall A, Lappas G. Risk factors of cardiovascular disease during the period 1985 - 1995 in Göteborg, Sweden. The GOT-MONICA Project. J Intern M ed 1997; 242:199-211.

22. Yao M, McCrory MA, Ma G, Li Y, Dolnikowski GG, SB Roberts. Energy requirements of urban Chinese adults with manual or sedentary occupations, determined using the doubly labeled water method. Eur J Clin Nutr 2002; 56:575-584.

23. Schofield WN. Predicting basal metabolic rate, new standards and review of previous work. Hum Nutr: Clin Nutr 1985; 39C(Suppl 1):5-41.

24. U.S. Department of $\mathrm{H}$ ealth and Human Services Physical Activity and $\mathrm{H}$ ealth. A Report of the Surgeon General. Atlanta, GA: DHHS, Centers for Disease Control and Prevention, National Center for Chronic Disease Prevention and Health Promotion; 1996.

25. Pate RR, Pratt M, Blair SN, Haskell WL, Macera $C A$, Bouchard $C$, Buchner $D$, Ettinger $W$, Heath GW, King AC, Kriska A, Leon AS, Marcus BH, M orris J, Paffenbarger RS Jr, Patrick K, Pollock ML, Rippe JM, Sallis J, Wilmore JH. Physical Activity and public health: A recommendation from the centers for disease control and prevention and the American College of Sports M edicine. JAM A 1995; 273:402-407
26. Coward WA. Contributions of the doubly labeled water method to studies of energy balance in the Third World. Am J Clin Nutr 1998; 68(Suppl):962S969S.

27. Barbosa TBC, Anjos LA, Vasconcellos M TL. Padrão de atividade física de adultos residentes no município de Niterói, Rio de Janeiro: Resultados da Pesquisa de Nutrição, Atividade Física e Saúde - PNAFS. [Submetido à aprovação].

28. Haskell WL, Lee IM, Pate RR, Powell KE, Blair SN, Franklin BA, Macera CA, Heath GW, Thompson $P D$, Bauman A. Physical activity and public health: updated recommendation for adults from the American College of Sports M edicine and the American Heart Association. Med Sci Sports Exerc 2007; 39(8):1423-1434.

29. Alfonzo-González $G$, Doucet $E$, Alméras N, Bouchard C, Tremblay A. Estimation of daily energy needs with the FAO/WHO/UNU 1985 procedures in adults: comparison to whole-body indirect calorimetry measurements. Eur J Clin Nutr 2004; 58:1125-1131.

30. Alemán-M ateo $H$, Salazar $G$, H ernández-Triana $M$, Valencia ME. Total energy expenditure, resting metabolic rate and physical activity level in free-living rural elderly men and women from Cuba, Chile and M exico. Eur J Clin Nutr 2006; 60:1258-1265.

31. Wahrlich V, Anjos LA. Aspectos históricos e metodológicos da medição e estimativa da taxa metabólica basal: U ma revisão da literatura. Cad Saude Publica 2001; 17(4):801-817.

32. Instituto Brasileiro de Geografia e Estatística. Pesquisa de Orçamentos Familiares - POF 2002 - 2003. [acessado 2006 Mai 31]. Disponível em: http:// www.ibge.gov.br/home/presidencia/noticias

33. Tooze JA, Schoeller DA, Subar AF, Kipnis V, Schatzkin A, Troiano RP. Total daily energy expenditure among middle-aged men and women: the OPEN Study. Am J Clin Nutr 2007; 86:382-387.

34. Vasconcelos MTL, Anjos LA. A simplified method for assessing physical activity level values for a country or study population. Eur J Clin N utr 2003; 57:1025-1033.

35. Anjos LA, Wahrlich V, Bossan FM, Salies M N, Silva $P B$. Energy expenditure of walking at different intensities in Brazilian college women. Clin Nutr 2008:27(1):121-125.

Artigo apresentado em 01/04/2008

Aprovado em 26/06/2008

Versão final apresentada em 08/07/2008 